\title{
Salud sexual en Chile: una aproximación descriptiva al comportamiento y la satisfacción sexual de los chilenos
}

\author{
Camila Mella S. ${ }^{1}$, Juan Carlos Oyanedel S. ${ }^{2}$, Salvador Vargas S. ${ }^{3}$, Nerea de Ugarte L. ${ }^{4}$ \\ ${ }^{1}$ Socióloga. Doctorante en Política Social, Universidad de Oxford. Investigadora Universidad de Santiago de Chile. ${ }^{2}$ So- \\ ciólogo. PhD in Law, King'sCollege London. Departamento de Gestión y Políticas Públicas, Facultad de Administración \\ y Economía, Universidad de Santiago de Chile. ${ }^{3}$ Sociólogo. Investigador Universidad de Santiago de Chile. ${ }^{4}$ Psicóloga. \\ Magíster en Psicología Clínica, Universidad Andrés Bello.
}

\section{RESUMEN}

Antecedentes: El año 2006, la Organización Mundial de la Salud definió a la "salud sexual" como un estado de bienestar físico, emocional, mental y social en relación a la propia sexualidad. De este modo, corresponde a un concepto más amplio que la ausencia de malestares, disfunciones o enfermedades, relacionándose con un nivel de bienestar general. Pese a la relevancia del concepto, aún es escasa la evidencia empírica al respecto a nivel local. Objetivos: El artículo analiza el concepto de "salud sexual" a nivel de población general en Chile. Métodos: Se diseñó una muestra probabilística, polietápica y estratificada en el Gran Santiago, encuestándose a 767 personas mayores de 18 años, siguiendo la metodología de autorreporte de hogares. Resultados: El estudio evidencia diferencias según género y edad en la mayor parte de las variables relativas a la satisfacción sexual y a conductas sexuales seguras. Conclusión: Se concluye que los hombres inician su vida sexual más tempranamente, tienen más parejas sexuales y reportan mayor satisfacción sexual que las mujeres, con excepción de la cohorte más joven. Ellas, a su vez, declaran mayores niveles de protección y cuidado de la vida sexual.

\section{PALABRAS CLAVES: Salud sexual, género, Chile}

\section{SUMMARY}

Background: In 2006, the World Health Organization endorsed the concept of "sexual health" as a state of physical, emotional, mental and social well-being in relation to sexuality. Thus, it corresponds to a broader concept that the absence of disease, dysfunction or infirmity, being related to a general level of well-being. Despite the relevance of the concept, locally, there is still scarce empirical evidence about it. Aims: The article analyses the concept of "sexual health" among the Chilean general population. Methods: In order to achieve this objective, a probabilistic, polyphasic and stratified sample was designed for the province of Santiago, being surveyed 767 people over 18 years, through a self-reported method in their own households. Results: The study shows differences by gender and age in both sexual satisfaction and safe sexual behaviours. Conclusion: It is possible to conclude that men begin their sexual life earlier, have more sexual partners and report higher sexual satisfaction than women, except for the youngest cohort. Women, in turn, report higher levels of protection and care of sexual life.

KEYWORDS: Sexual health, gender, Chile 


\section{INTRODUCCIÓN}

A partir del año 2006, la Organización Mundial de la Salud ha definido el concepto de "salud sexual" en términos de dos principales componentes: por un lado, como un nivel de bienestar general a nivel emocional, mental, físico y social respecto a la forma de vivir y experimentar la propia sexualidad. Por otro lado, en relación a mantener prácticas sexuales seguras, que permitan a los individuos vivir su sexualidad en ausencia de potenciales problemas físicos, enfermedades o disfunciones de índole sexual (1).

En esta materia, existe escasa evidencia empírica a nivel local; especialmente en temas de satisfacción sexual para el caso de Chile. Al respecto, el estudio de Barrientos y Páez (2) es una excepción destacable. Utilizando datos de la Encuesta "Comportamiento Sexual del Cono Sur" (COSECON) del año 1998, los autores determinaron que el 51,8\% de los hombres se encuentran satisfechos con sus encuentros sexuales, descendiendo esa cifra hasta el $44,4 \%$ para el caso de las mujeres. Posteriormente, el Programa de las Naciones Unidas para el Desarrollo (PNUD) elaboró datos que incluyeron medidas de satisfacción y comportamiento sexual para el Informe de Desarrollo Humano del año 2012 (3), determinando un promedio de 7,76 $\pm 2,16$ para la satisfacción sexual entre hombres, y de 6,98 \pm 2,75 para mujeres, utilizando una escala que oscila entre 1 y 10 puntos.

La literatura internacional es controversial respecto de las diferencias por género respecto de la satisfacción sexual (4). Sin embargo, en la década de 1970, estudios longitudinales sugieren que existiría un efecto generacional asociado, en la medida que la brecha entre hombres y mujeres era mayor entonces que en la actualidad (5). A su vez, otras investigaciones han analizado la interacción entre sexo y edad, estableciendo que los puntajes son similares; sin embargo, en hombres tienden a ser más estables a lo largo del ciclo vital mientras que las mujeres comienzan a descender luego de los 45 años (6), así como también a disminuir la frecuencia de relaciones sexuales (7).

En lo referente a la seguridad asociada a las relaciones sexuales, en Chile las investigaciones sostienen que el inicio de la vida sexual de los y las jóvenes está asociado con una escasa protección (8), siendo irregular el uso de métodos de barrera en este grupo etario (9). Los motivos para el uso del preservativo masculino tienden a estar relacionados con la percepción de riesgo con infecciones de transmisión sexual (10), lo que puede llevar a que su utilización disminuya a medida que las relaciones de pareja se mantienen en el tiempo y siendo reemplazados por métodos hormonales, tal como ha destacado la literatura internacional $(11,12)$.
Considerando estos antecedentes y, en particular, la falta de estudios en el área para el caso de Chile, se realizó la Primera Encuesta sobre Satisfacción, Deseo y Satisfacción Sexual en Chile, durante los meses de agosto y noviembre de 2014. Recogiendo la información producida en este estudio, el artículo tiene como objetivo describir los componentes de la salud sexual en una muestra representativa de la Región Metropolitana, considerando a la edad y el sexo como variables claves.

\section{SUJETOS Y MÉTODO}

La "Encuesta sobre Comportamiento, Deseo y Satisfacción Sexual" corresponde a una iniciativa desarrollada por el equipo de investigación del Centro de Estudios Cuantitativos de la Facultad de Administración y Economía de la Universidad de Santiago de Chile. Al respecto, cabe precisar que corresponde a una iniciativa pionera debido a que evalúa estas materias en población no clínica, aplicando una metodología innovadora: simultáneamente se realizó una encuesta online (la cual fue difundida a través de distintos medios de comunicación) y otra de autorreporte de hogares. Es necesario señalar que el presente artículo consigna solamente los resultados obtenidos en la versión de hogares, marcando además, el inicio de la divulgación parcial de los resultados generales del estudio.

Para la encuesta de hogares se diseñó una muestra probabilística, polietápica y estratificada en el Gran Santiago, el cual corresponde a las zonas urbanas de la provincia de Santiago, además de Puente Alto y San Bernardo. El diseño muestral se elaboró sobre la base de la información del Censo de Población y Vivienda del año 2002 (marco muestral del Instituto Nacional de Estadísticas), y consideró tres unidades muestrales principales:

La unidad de muestreo primaria correspondió a los estratos, definidos como la inclusión forzosa de las 32 comunas urbanas del Gran Santiago, con distribución de casos según nivel socioeconómico (ABC1, C2, C3, D), con afijación igual.

La unidad de muestreo secundaria correspondió a las manzanas, seleccionadas aleatoriamente dentro de cada comuna, con probabilidad de selección igual dentro de cada estrato (una manzana por estrato).

La unidad de muestreo terciaria correspondió a las viviendas, siendo seleccionadas 10 en cada manzana según distribución de la tabla de Kish para asegurar la aleatoriedad en su selección. Una vez en la vivienda, se seleccionó sólo un hogar.

La población objetivo consideró a las personas mayores de 18 años y que, a lo menos, declarasen haber tenido una relación sexual con penetración durante su vida. Cabe señalar que por hogar seleccionado se encuestó a sólo un/a residente que cumpliese con este criterio. 
Considerando las temáticas abordadas, la encuesta siguió un formato de autorreporte de hogares, de modo tal de evitar la posible influencia del entrevistador en las respuesta de las personas. Para ello, se tomaron las siguientes consideraciones:

En primer lugar, todos los encuestadores debieron asistir a una capacitación de carácter presencial y obligatorio, en donde se especificaron: los objetivos del estudio, cada una de las preguntas del cuestionario, y la metodología del levantamiento (incluyendo el manejo de hojas de ruta y aleatoriedad de los entrevistados).

En segundo lugar, cada una de las encuestas aplicadas contó con un consentimiento informado, en donde se explicitaron los objetivos del estudio, uso de la información recopilada, y datos de contacto de los investigadores responsables. Es importante detallar que cada encuesta debía contar con dicho consentimiento firmado. En caso de no ser así, el material no era considerado válido, siendo excluido de la base de datos.

En tercer lugar, la metodología de autorreporte se aplicó de acuerdo al siguiente procedimiento: una vez que el entrevistado aceptaba participar del estudio y firmaba el consentimiento informado, el cuestionario era dejado con él / ella para que lo respondiese de forma individual. Como insumo, también se dejó un sobre, en el cual el entrevistado debía devolver sellado con la encuesta respondida en su interior. Para ello, al momento de firmar el consentimiento informado se acordaba un tiempo de devolución con el entrevistado (el tiempo sugerido por el equipo de investigación fue de 48 horas).

La evaluación del material, construcción y validación de la base de datos se efectuó de manera centralizada en dependencias del Centro de Estudios Cuantitativos. Para ello, se habilitó una plataforma de digitación online, diseñada especialmente para efectos de esta investigación. De la base de datos fueron excluidos los casos que: 1) que tuvieran el folio (identificador del cuestionario) duplicado (para lo cual se cotejó cuál de las dos versiones correspondía a la versión en papel); 2) no contaran con consentimiento informado firmado y validado por el supervisor de terreno; 3) tuvieran información sociodemográfica incompleta que no permitiese la ponderación de los datos; y 4) no contaran con, al menos, el $75 \%$ de las respuestas completas.
Siguiendo las especificaciones previas, el levantamiento alcanzó una muestra total de 767 casos, de los cuales el 45,9\% correspondió a hombres, y un $54,1 \%$ a mujeres; con una edad promedio de $42,31 \pm 16,74$ años. El error asociado a la muestra es de $3,54 \%$, con un nivel de confianza del $95 \%$. Los datos fueron ponderados por sexo, edad y nivel socioeconómico.

Específicamente, para efectos del presente artículo, los resultados fueron segmentados según sexo y tramo etario. Asimismo, en relación a la satisfacción sexual, se consideraron sólo las preguntas concernientes a: frecuencia de relaciones sexuales, forma más habitual de ocurrencia de un orgasmo, número de parejas sexuales a lo largo de la vida, edad de primera relación sexual con penetración, y satisfacción respecto a su vida sexual. Por su parte, al indagar respecto a las prácticas sexuales seguras, se consideró la utilización de métodos anticonceptivos (por parte del/a entrevistado/a y su pareja) durante la última relación sexual con penetración.

Los datos fueron analizados a través del software estadístico IBM-SPSS, v. 20.

\section{RESULTADOS}

Se observa que la edad de la primera relación sexual es menor en todos los tramos etarios entre los hombres, sin evidenciarse un descenso en las cohortes más jóvenes como sucede en el caso de las mujeres. Así, las entrevistadas mayores de 56 años presentan una edad media de 23,93 $\pm 18,53$ años, el grupo que abarca 46 a 55 años de 18,89 \pm 4,23 años, en el rango de 31 a 45 años de 17,72 \pm 3,32 años, y finalmente, en el tramo entre 18 y 30 años presentan un promedio de 16,38 $\pm 2,36$ años (Tabla I).

Al indagar en el número de parejas sexuales que se han tenido a lo largo de la vida, los entrevistados de todos los tramos etarios presentan medias más altas que sus pares mujeres. Entre ellas, los promedios tienden a ser estables, en torno a 4 parejas, descendiendo entre las mayores de 56 años a una media de $2,75 \pm 2,89$ parejas. Por el contrario, entre los hombres, la misma cohorte presenta el mayor promedio de parejas, alcanzando una media de $15,11 \pm 16,37$ parejas (Tabla II).

\section{Tabla I \\ EDAD DE LA PRIMERA RELACIÓN SEXUAL}

\begin{tabular}{|c|c|c|c|c|c|c|c|c|}
\hline & \multicolumn{2}{|c|}{ 18-30 años } & \multicolumn{2}{|c|}{ 31-45 años } & \multicolumn{2}{|c|}{ 46-55 años } & \multicolumn{2}{|c|}{$\geq 56$ años } \\
\hline & Media & $\pm \mathrm{DE}$ & Media & $\pm \mathrm{DE}$ & Media & $\pm \mathrm{DE}$ & Media & $\pm \mathrm{DE}$ \\
\hline Hombre & 15,39 & 2,819 & 15,69 & 3,507 & 16,43 & 6,638 & 15,14 & 2,962 \\
\hline Mujer & 16,38 & 2,365 & 17,72 & 3,324 & 18,89 & 4,239 & 23,93 & 18,535 \\
\hline
\end{tabular}




\section{NÚMERO DE PAREJAS SEXUALES}

\begin{tabular}{|c|c|c|c|c|c|c|c|c|}
\hline & \multicolumn{2}{|c|}{ 18-30 años } & \multicolumn{2}{|c|}{ 31-45 años } & \multicolumn{2}{|c|}{ 46-55 años } & \multicolumn{2}{|c|}{$\geq 56$ años } \\
\hline & Media & $\pm \mathrm{DE}$ & Media & $\pm \mathrm{DE}$ & Media & $\pm \mathrm{DE}$ & Media & $\pm \mathrm{DE}$ \\
\hline Hombre & 6,06 & 6,872 & 12,38 & 15,990 & 10,33 & 14,147 & 15,11 & 16,379 \\
\hline Mujer & 4,07 & 4,864 & 4,11 & 3,722 & 4,30 & 4,184 & 2,75 & 2,892 \\
\hline
\end{tabular}

Respecto de la forma más habitual de tener un orgasmo, es destacable, en primer término, que sólo el $2,7 \%$ de los hombres del rango entre 18 y 30 años declara nunca haber tenido uno, mientras que no se observan frecuencias en esta categoría en los restantes tramos etarios. Esto contrasta con lo reportado por las mujeres, dado que el $6,4 \%$ de las más jóvenes nunca ha tenido un orgasmo, tal como el $10,2 \%$ de las mayores de 56 años. Sin embargo, para ambos géneros, la práctica que con mayor frecuencia es declarada como medio principal para alcanzar un orgasmo es la penetración vaginal. En efecto, entre las cohortes más jóvenes dicha práctica es mencionada más frecuentemente por los hombres; mientras que, en el caso de las mujeres, destaca el alto porcentaje reportado entre las mayores de 46 años. Mientras tanto, en todos los cruces entre sexo y edad, la segunda práctica en importancia respecto de obtener un orgasmo es recibir sexo oral, especialmente entre los hombres mayores de 56 años (26,8\%) (Tabla III).

Respecto a la frecuencia de mantener encuentros sexuales durante el último año, la pregunta era de tipo ordinal, presentando como categorías de respuesta: "no he tenido relaciones sexuales", "menos de una vez al mes", "una vez al mes", "dos veces al mes", "una vez a la semana"y "más de una vez a la semana", asignándose puntuaciones entre 0 y 5 , respectivamente. De este modo, la primera cifra representa la frecuencia nula y el 5 , la de mayor ocurrencia. Al comparar según género, los hombres reportan una mayor frecuencia de mantener relaciones sexuales, siendo el tramo de 46 a 55 años el que alcanza una media más alta, con 4,10 $\pm 1,21$ puntos. La excepción la constituye la cohorte más joven, donde las mujeres presentan una media de 3,29 $\pm 1,77$ puntos (Tabla IV).

Tabla III

FORMAS HABITUALES DE LOGRAR ORGASMO

\begin{tabular}{lcccc}
\hline & $18-30$ años & $31-45$ años & $46-55$ años & $\geq 56$ años \\
\hline Hombres (\%) & & & & \\
\hline Nunca he tenido un orgasmo & 2,7 & 0,0 & 0,0 & 26,0 \\
Recibir sexo oral & 15,4 & 16,1 & 76,1 & 72,3 \\
Penetración vaginal & 69,3 & 67,2 & 1,2 & 0,8 \\
Penetración anal & 6,4 & 12,4 & 0,0 & 0,0 \\
Masturbación & 5,5 & 2,7 & 0,0 & 0,0 \\
Que su pareja lo/a masturbe & 0,7 & 1,6 & & 1,8 \\
\hline Mujeres (\%) & & & 10,7 & 0,2 \\
\hline Nunca he tenido un orgasmo & 6,4 & 2,5 & 77,4 & 82,4 \\
Recibir sexo oral & 19,1 & 17,4 & 0,0 & 0,0 \\
Penetración vaginal & 56,6 & 62,1 & 5,1 & 1,1 \\
Penetración anal & 4,8 & 0,7 & 5,0 & 6,4 \\
Masturbación & 6,6 & 9,5 & & \\
Que su pareja lo/a masturbe & 6,5 & & & \\
\hline
\end{tabular}




\section{FRECUENCIA DE RELACIONES SEXUALES}

\begin{tabular}{|c|c|c|c|c|c|c|c|c|}
\hline & \multicolumn{2}{|c|}{ 18-30 años } & \multicolumn{2}{|c|}{ 31-45 años } & \multicolumn{2}{|c|}{ 46-55 años } & \multicolumn{2}{|c|}{$\geq 56$ años } \\
\hline & Media & $\pm \mathrm{DE}$ & Media & $\pm \mathrm{DE}$ & Media & $\pm \mathrm{DE}$ & Media & $\pm \mathrm{DE}$ \\
\hline Hombre & 3,23 & 1,884 & 3,81 & 1,387 & 4,10 & 1,218 & 3,11 & 1,806 \\
\hline Mujer & 3,29 & 1,771 & 3,24 & 1,780 & 3,08 & 1,782 & 1,04 & 1,417 \\
\hline
\end{tabular}

Un comportamiento similar a la frecuencia de relaciones sexuales presenta la satisfacción con la vida sexual, medida en una escala que oscila entre 0 y 10 puntos, donde la primera cifra representa "completamente insatisfecho" y la segunda, "completamente satisfecho". Las mujeres presentan el promedio más alto para el grupo más joven, con una media de 7,90 $\pm 2,73$ puntos. Sin embargo, en las cohortes restantes, son los hombres quienes declaran mayor satisfacción en relación a su vida sexual, alcanzando la media más alta del estudio el tramo cuya edad oscila entre los 46 y 55 años (con un promedio de $7,77 \pm 2,10$ puntos) (Tabla V).

Respecto al uso de anticonceptivos, se observa que entre las mujeres el método más importante varía según la edad. De este modo, los más importantes para el grupo entre 18 y 30 años son los métodos orales $(51,5 \%)$, disminuyendo su uso reportado para la cohorte siguiente, y siendo desplazado por el dispositivo intrauterino entre las mayores de 46 años (25,8\%). Para los hombres, en tanto, el condón corresponde al método predominante, lo que se debe a que la alternativa restante -la vasectomía- presenta frecuencias muy bajas. Otro elemento importante a considerar es el porcentaje que declara no utilizar ningún método anticonceptivo, el cual es superior en los hombres para todos los tramos etarios. En particular, destaca que el $15,2 \%$ de las mujeres y el 39,0\% de los hombres entre 18 y 30 años señalan no haber utilizado ninguna alternativa anticonceptiva durante su última relación sexual penetrativa (Tabla VI).

\section{Tabla V}

SATISFACCIÓN CON LA VIDA SEXUAL

\begin{tabular}{lcccccccr}
\hline & \multicolumn{2}{c}{$18-30$ años } & \multicolumn{2}{c}{$31-45$ años } & \multicolumn{2}{c}{$46-55$ años } & \multicolumn{2}{c}{$\geq 56$ años } \\
& Media & \pm DE & Media & \pm DE & Media & \pm DE & Media & \pm DE \\
\hline Hombre & 7,78 & 2,698 & 7,57 & 2,604 & 7,77 & 2,103 & 7,60 & 2,666 \\
Mujer & 7,90 & 2,739 & 7,31 & 2,751 & 6,68 & 3,452 & 5,45 & 3,382 \\
\hline
\end{tabular}

Tabla VI

USO DE MÉTODOS ANTICONCEPTIVOS

\begin{tabular}{llcccc}
\hline & Frecuencia (\%) & 18-30 años & 31-45 años & 46-55 años & $\geq 56$ años \\
\hline \multirow{4}{*}{ Mujer } & Anticoncepción oral & 51,5 & 40,5 & 15,7 & 9,5 \\
& Dispositivo intrauterino & 4,7 & 14,9 & 25,8 & 23,1 \\
& Inyectable & 12,7 & 9,9 & 5,8 & 0,0 \\
& Esterilización quirúrgica & 0,8 & 12,8 & 11,9 & 39,9 \\
& Otro & 15,1 & 10,6 & 6,5 & 0,0 \\
& Ninguna & 15,2 & 11,3 & 33,8 & 28,1 \\
\hline \multirow{3}{*}{ Hombre } & Condón & 61,0 & 54,3 & 35,6 & 18,4 \\
& Vasectomía & 0,0 & 2,7 & 0,0 & 5,2 \\
& Ninguna & 39,0 & 43,0 & 64,4 & 76,4 \\
\hline
\end{tabular}


A partir de lo anterior, de los encuestados que declararon no utilizar ningún método anticonceptivo, son los hombres quienes mayormente reportan que su pareja tampoco utilizó ningún tipo de método de barrera durante la última relación sexual, proporción que también aumenta con la edad. De este modo, es posible señalar que las conductas de protección sexual, principalmente, recaen sobre las mujeres: por ejemplo, para la cohorte entre 18 y 30 años, sólo el $36,2 \%$ de los hombres reporta que sus parejas sexuales no utilizaron alguna alternativa de barrera durante la última relación sexual, al mismo tiempo que el $60,5 \%$ de las mujeres en el mismo tramo (Tabla VII).

\section{DISCUSIÓN}

Los resultados presentados dan cuenta de importantes diferencias según género y tramos etarios en relación al comportamiento y a la satisfacción sexual. De este modo, en relación a lo primero, los hombres reportan un inicio sexual más precoz que sus pares mujeres en todos los tramos etarios, siendo las diferencias más marcadas en la cohorte de mayor edad en donde ellas indican que la iniciación de su vida sexual fue después de los 20 años. A su vez, los hombres también reportan un mayor número de parejas sexuales a lo largo de su vida que las mujeres en todos los tramos etarios. En este aspecto, asimismo, destaca que las cohortes mayores poseen un menor número de parejas sexuales que los más jóvenes, para ambos géneros. Estos resultados siguen la tendencia descrita por otros estudios en la materia $(13,14)$, destacando que las personas de edad más avanzada sí tienen vida sexual activa.

En relación a lo segundo, la satisfacción sexual tiende a ser mayor en los hombres, exceptuando a la cohorte más joven en donde las mujeres se declaran más satisfechas que sus pares de la misma edad. Estos resultados son coherentes con la evidencia de estudios internacionales (15), aumentando la brecha de satisfacción entre los entrevistados de mayor edad (16). En relación a ello, destaca que los datos también muestran que un porcentaje importante de mujeres que nunca ha tenido un orgasmo (6,4\% del grupo entre 18 y 30 años, y un $10,2 \%$ entre las mayores de 56 años).

Un punto de particular importancia radica en la responsabilidad asociada a los comportamientos sexuales. Tal como se evidenció, la definición de salud sexual de la Organización Mundial de la Salud (1), implica que la actividad sexual de las personas esté asociada no sólo al bienestar emocional y físico, sino también a la seguridad percibida en los encuentros sexuales. De este modo, es relevante que el $15,2 \%$ de las mujeres de 18 a 30 años no utilizó ningún método anticonceptivo durante su última relación sexual, mientras que el $39,0 \%$ de sus pares hombres de la misma edad señala lo mismo. Además, para la misma cohorte, el $36,2 \%$ de los hombres y el $60,5 \%$ de las mujeres sostienen que su pareja sexual no usó ningún método de barrera en el último encuentro sexual. Lo anterior, abre la posibilidad que se está incurriendo en prácticas sexuales de riesgo que, potencialmente, pueden derivar en contagios de infecciones de transmisión sexual y/o embarazos no deseados.

A partir de lo anterior, la literatura internacional sugiere que, entre los más jóvenes, la percepción sobre los métodos de protección está más enfocada hacia la prevención del embarazo y no de enfermedades de transmisión sexual $(17,18,19)$, lo cual debe ser considerado en el diseño de políticas públicas en la materia. Adicionalmente, es necesario precisar que el uso de anticoncepción presenta un marcado sesgo (o desbalance) por género respecto al cuidado en las relaciones sexuales. Por su parte, la literatura nacional señala que la principal razón para no utilizar anticoncepción es creer que "no se necesita", y no por motivos de salud, falta de acceso u oposición a su uso (20). En efecto, el repertorio de métodos de barrera más utilizados en Chile corresponden a los dispositivos intrauterinos, pastillas anticonceptivas o esterilización femenina, variando la prioridad de su uso de acuerdo al

\section{Tabla VII \\ USO DE MÉTODOS ANTICONCEPTIVOS POR PARTE DE LA PAREJA ENTRE QUIENES DECLARAN NO UTILIZAR ALGUNO}

\begin{tabular}{lrccrrr}
\hline & & $18-30$ años & $31-45$ años & $46-55$ años & $\geq 56$ años \\
\hline \multirow{2}{*}{ Hombre (\%) } & Sí & 63,8 & 44,5 & 32,2 & 6,9 \\
& No & 36,2 & 55,5 & 67,8 & 93,1 \\
\hline \multirow{2}{*}{ Mujer (\%) } & Sí & 39,5 & 2,2 & 22,0 & 0,0 \\
& No & 60,5 & 97,8 & 78,0 & 100,0 \\
\hline
\end{tabular}


tramo etario (20). Algunos factores que explicarían este fenómeno se vinculan al rol que juegan los hombres en las decisiones respecto a los métodos anticonceptivos, específicamente, en la promoción del uso de alternativas menos invasivas para ellos (21). Dicha situación puede responder al rol preponderante que juegan los hombres en materia de intimidad emocional (21) o por el papel dominante que juega el placer sexual masculino en sociedades androcéntricas como las latinoamericanas (22).

Ahora bien, los resultados presentados deben ser considerados con precaución en la medida en que corresponden a un primer acercamiento hacia la producción de información en materia de salud sexual en Chile. En este sentido, son una aproximación descriptiva en temas de comportamiento y satisfacción sexual, sin evaluar posibles efectos explicativos ni longitudinales al respecto. No obstante, es necesario precisar que las diferencias reportadas según género y edad entregan información útil para el diseño de políticas públicas en salud sexual, por ejemplo: en el diseño de estrategias de prevención considerando el bajo uso de preservativos en el caso de los hombres, propiciar la responsabilidad compartida en materia de anticoncepción y prevención de contagio de enfermedades de transmisión sexual, la concientización en torno a los derechos sexuales y reproductivos en el caso de las mujeres, y relevar el rol de la satisfacción sexual, entre otros.

\section{CONCLUSIÓN}

Es posible concluir que en materia de salud sexual existen diferencias según género y edad. De este modo, destaca que los hombres reportan mayores niveles de satisfacción sexual; declarando, además de un inicio sexual más precoz, un mayor número de parejas sexuales, y un uso menos frecuente de métodos anticonceptivos o de barrera. En contraposición, las mujeres declaran menores niveles de satisfacción sexual en relación a sus pares masculinos, con excepción de la cohorte entre 18 y 30 años; además de un aumento del número de parejas sexuales en las generaciones más jóvenes, y de ser quienes se encargan de la seguridad de las relaciones sexuales al reportar un uso más frecuente de anticoncepción.

\section{REFERENCIAS}

1. World Health Organization. Defining sexual health. Report of a technical consultation on sexual health. pp 28-31; January 2002. Geneva: World Health Organization, 2006.

2. Barrientos J, Páez D. Psychosocial Variables of Sexual Satisfaction in Chile. J Sex Marital Ther. 2006;3(3):351-68.
3. Programa de las Naciones Unidas para el Desarrollo. Desarrollo humano en Chile 2012. Bienestar subjetivo: el desafío de repensar el desarrollo, Santiago: Programa de las Naciones Unidas para el Desarrollo, 2012.

4. Sánchez M, Santos P, Sierra J. A systematic review of sexual satisfaction. Inter J Clin Health Psychology. 2014;14(1):67-75.

5. Haavio E, Kontula O. Correlates of Increased Sexual Satisfaction. Arch Sex Behav. 1997;26(4):399-419.

6. Castellanos E, Álvarez C, Ruiz D, Pérez G. Social determinants of sexual satisfaction in Spain considered from the gender perspective. Ann Epidemiol. 2013;23(3):150-6.

7. Dennerstein L, Lehert P, Burger H, Dudley E. Factors affecting sexual functioning of women in the mid-life years. Climateric. 1999;2(4):254-62.

8. Montero A, González E, Molina T. Salud sexual y reproductiva de los y las adolescentes en Chile. Rev Chil Salud Pública. 2008;12(1):42-7.

9. González E, Luengo X, Sandoval J, Molina T, Caba F. Estudio comparativo de factores familiares y personales en adolescentes consultantes por embarazo y anticoncepción. Rev Chil Obstet Ginecol Infantil Adolescencia. 1998;5(2):42-8.

10. Wei L. A comparison of consensus and non-consensus approaches to modelling contraceptive behaviour. Health Econ. 2002;(11):599-622.

11. Wildsmith E, Manlove J, Steward N. Relationship characteristics and contraceptive use among dating and cohabiting young adult couples. Perspect Sex Reprod Health. 2015;47(1):27-36.

12. Manlove J, Welti K, Wildsmith E, Barry M. Relationship types and contraceptive use within young adult dating relationships. Perspect Sex Reprod Health. 2014;46(1):41-50.

13. Wolowski A, Oliel $Y$, Leefsma M, Drorith $H$. Sexual activities, sexual and life satisfaction, and successful aging in women. J Sex Med. 2010;7(7):2401-10.

14. Silva JM, Ramírez D. Comportamiento sexual en la población mayor de 70 años. Urología Colombiana. 2006;15:71-6.

15. Navarro B, Ros L, Latorre JM, Escribano C, López V, Romero M. Hábitos, Preferencias y Satisfacción Sexual en Estudiantes Universitarios. Revista Clínica de Medicina de Familia. 2010;3(3):150-7.

16. González JM, González J. Estudio descriptivo de la sexualidad de personas mayores de 60 años en el Caribe Colombiano. Psicogente. 2005;(13):17-27.

17. Duberstein L, Ku L, Sonenstein F. Adolescents males' combined use of condoms with partners' use of female contraceptive methods. Matern Child Health $\mathrm{J}$. 1998;2(4):201-9.

18. Chirinos J, Bardales O, Segura M. Las relaciones coitales y la percepción de riesgo de adquirir ETS/SIDA en adultos jóvenes varones de Lima, Perú. Cad. Saúde Pública. 2006;22(1):79-85.

19 Vasilenko S, Kreager D, Lefkowitz E. Gender, contraceptive attitudes, and condom use in adolescent romanti relationships: a dyadic approach. J Res Adolesc. 2015;25(1):51-62.

20 Encuesta Nacional de Calidad de Vida y Salud, 2000. Informe de Planificación Familiar, Módulo II. Hallado 
en: http://epi.minsal.cl/wp-content/uploads/2012/07/ infplanfam.pdf. Acceso: 16 de julio de 2015

21. Tschann J, Adler N, Millstein S, Gurvey J, Ellen J. Relative power between sexual partners and condom use among adolescents. J Adolesc Health. 2002;31(1):1725.
22 Spiegelhalter, D. Sex by numbers: what statistics can tell us about sexual behaviour. Wellcome Collection, 2015. 